\title{
Management of Idiopathic Anaphylaxis: When Is Bone Marrow Examination Essential?
}

Jim Fernandez, MD, $P h D^{1,}$ Cem Akin, MD, $P h D^{2, *}$

\section{Address}

Cleveland Clinic Foundation, 9500 Euclid Ave, Cleveland, OH 44195, USA

*Brigham \& Women's Hospital, 1 Jimmy Fund Way, Boston, MA 02115, USA

Email: cakin@partners.org

Published online: 13 May 2014

(C) Springer International Publishing AG 2014

Keywords Anaphylaxis - Mastocytosis - Mast cell activation - Angioedema - Tryptase - Histamine - C-kit - Bone marrow biopsy · Epinephrine · Urticaria pigmentosa

\section{Opinion statement}

Idiopathic anaphylaxis is not an uncommon entity within the Allergy and Immunology field. Management of such patients can be extremely challenging at times. A thorough history, including an emphasis on possible mast cell disease, is an absolute necessity. A bone marrow biopsy is not only appropriate, but essential, when evaluating patients with recurrent anaphylaxis if a clonal mast cell disease is suspected as the underlying etiology. Clinical predictors for underlying clonal mast cell disease have been studied and include an elevated baseline tryptase, presence of hypotensive syncopal episodes, and absence of urticaria and angioedema during anaphylactic episodes. In the acute setting, there is no alternative to self-injectable epinephrine for treatment of suspected anaphylaxis of any type. It should be administered with repeated doses as deemed necessary to establish resolution of symptoms such as hypotension, bronchospasm, or cardiovascular collapse. Once the diagnosis of idiopathic anaphylaxis is made, we recommend starting maintenance therapy with a regimen of $\mathrm{H} 1$ antihistamines. Cases who are refractory to $\mathrm{H} 1$ antihistamine therapy alone will require the addition of at least low dose immune suppression with corticosteroids to provide continued relief from recurrent episodes. In many cases these medications can be weaned in less than a year without recurrent symptoms. In refractory cases or corticosteroid-dependent idiopathic anaphylaxis, adjunctive therapy with second line agents including montelukast or omalizumab has been used with some success. 


\section{Introduction}

Anaphylaxis is defined as a serious allergic reaction that is rapid in onset and may cause death. Although there are a variety of triggers that have been documented to cause anaphylaxis, the culprit is frequently not identifiable in patients with multiple recurrent episodes. These patients are diagnosed with idiopathic anaphylaxis. The symptoms of idiopathic anaphylaxis are not unique to other forms of anaphylaxis and a diagnosis is made after all other possible triggers have been eliminated. In many of these cases, other diseases such as mastocytosis, mast cell activation syndrome, angioedema, carcinoid syndrome, and vocal cord dysfunction need to be investigated and ruled out prior to making the diagnosis of idiopathic anaphylaxis. Current treatment focuses on acute and prophylactic measures to protect against severe or even life threatening events.

\section{Epidemiology}

Idiopathic anaphylaxis can occur at any age but is more common in adults than children [1]. The actual prevalence of idiopathic anaphylaxis is difficult to determine but is has been estimated that there have been as many as 40,000 reported cases in the past 30 years [2]. Studies also show that in at least one third of all anaphylactic episodes, no identifiable trigger is identified [3]. Risk factors for idiopathic anaphylaxis appear to be: female gender; atopy; a history of anaphylaxis with an identifiable trigger; and food allergy [4].

\section{Pathogenesis}

Idiopathic anaphylaxis was first described in 1978 [5], and since that time a few clues to the pathogenesis of the disease have been uncovered. Over the years it has been noted to be a steroid sensitive disease, which many argue for an underlying autoimmune process. Previous studies have identified a higher percentage of activated T cells (CD3+HLA-DR+cells) in the blood of patients with idiopathic anaphylaxis compared to the normal population (Lymphocyte subsets and activation markers in patients with acute episodes of idiopathic anaphylaxis) [6]. The exact clinical significance of this finding is, however, not currently completely understood.

There is a known association with mast cell activation and idiopathic anaphylaxis [7]. Measurement of serum mast cell tryptase levels can help prove mast cell activation, and aid in the diagnosis of anaphylaxis, and differentiation from other syndromes presenting with similar symptoms. Patients with mastocytosis as well as idiopathic anaphylaxis have symptoms related to increased mast cell mediator release. These mediators include histamine, tryptase, prostaglandin D2, cysteinyl leukotrienes, and a variety of cytokines including TNF [8]. The end result of mediator release can affect multiple organs and may variably result in: flushing, pruritus, hives, angioedema, GI disturbances, hypotension, tachycardia, syncope, and cardiovascular collapse. Recurrent anaphylaxis is common in mastocytosis [9], and up to $40 \%$ of patients diagnosed with idiopathic anaphylaxis may have mastocytosis or a clonal mast cell disease, as the underlying diagnosis [7]. Thus there is a 
strong belief that mast cell degranulation plays a major role in idiopathic anaphylaxis [9].

\section{Presentation}

Signs and symptoms in patients with idiopathic anaphylaxis do not differ from patients with other forms of anaphylaxis, and can include hypotension, tachycardia, wheezing, stridor, hives, angioedema, pruritus, flushing, nausea, vomiting, diarrhea, dysphagia, light-headedness, and loss of consciousness. In one review of 335 patients with idiopathic anaphylaxis, all of them experienced hives and angioedema, whereas $60 \%$ experienced symptoms of upper airway obstruction [1]. Life threatening cardiovascular collapse can occur with or without cutaneous or other warning signs. Patients with similar symptoms related to mastocytosis or mast cell disease tend to lack urticaria $[10,11]$.

\section{Diagnosis}

By definition idiopathic anaphylaxis is a diagnosis of exclusion. A detailed history is an extremely important part of every patient's evaluation. The history should focus on the events surrounding each episode of anaphylaxis: the frequency and severity of attacks; other associated symptoms such as urticaria and angioedema; treatments attempted; response to treatment, and any other objective data (such as serum tryptase measurements during the acute episodes) that can be gathered from the patient or medical record. Known causes of anaphylaxis should be considered in every patient. These may include food, medications, insect stings, latex, radiographic contrast media, and exercise. Hereditary and acquired angioedema should also be evaluated with appropriate complement studies. Hereditary angioedema can be confused with anaphylactic episodes in certain settings. Urticaria is absent in patients with $\mathrm{HAE}$, and the episodic swelling tends to be quicker in HAE than angioedema associated with idiopathic anaphylaxis [12]. A positive family history of angioedema or swelling with surgeries or trauma can help distinction the etiology. The diagnosis can be evaluated by checking the levels of $\mathrm{C} 4, \mathrm{CH} 50$, and $\mathrm{C} 1$ esterase inhibitor concentration or function.

Clonal mast cell disease (i.e., systemic mastocytosis or monoclonal mast cell activation syndrome) may present with similar symptoms as idiopathic anaphylaxis. However, in contrast to IA, patients with clonal mast cell disease do not usually have accompanying urticaria or angioedema $[10,11]$. Many patients with mastocytosis have the unique cutaneous finding of urticaria pigmentosa, classically described as salmoncolored macules that urticate upon stroking (Darier's sign). In addition to the skin, mast cell infiltration may occur in the bone marrow, liver, spleen, and the gastrointestinal tract. Laboratory studies that may help diagnose mast cell disease include a baseline serum tryptase level, and D $816 \mathrm{~V}$ c-kit mutation; however the definitive diagnosis is established by a tissue biopsy (skin or bone marrow). 
When is a bone marrow biopsy necessary?

Evaluation for a clonal mast cell disease in patients with anaphylaxis is an important diagnostic tool to consider. One study examining 12 patients who were previously given a diagnosis of idiopathic anaphylaxis noted that five of the 12 patients had evidence of clonal mast cells in the bone marrow [7]. Of these, three had a D816V c-kit mutation and all had aberrant CD25 expression and as such, these patients met at least one criterion for systemic mastocytosis. In addition, a tryptase-stained bone marrow biopsy from these patients showed evidence of morphologically aberrant mast cells, such as those with elongated shapes and hypogranulation. Two patients had a baseline serum tryptase levels greater than $20 \mathrm{ng} / \mathrm{mL}$ [7]. None of the patients had urticaria pigmentosa skin lesions. It was noteworthy that all of the patients in this study were diagnosed as having idiopathic anaphylaxis prior to study entry, with some showing bone marrow examinations done elsewhere reporting no evidence of mastocytosis. Therefore, a bone marrow examination showing "normal" results does not completely rule out the presence of clonal mast cell disease, unless it is critically reviewed by an expert pathologist with experience in diagnosing mast cell disorders. It is critical that appropriate diagnostic studies on these bone marrows are performed. These include mast cell specific immunohistochemical stains of tryptase and CD117, as well as markers of mast cell clonality including CD25 and c-kit D816V mutation. It is not unusual to see no major mast cell clustering in bone marrows of patients with normal or only slightly elevated tryptase levels, however these tissue sections should be carefully evaluated for the presence of minor WHO diagnostic criteria. In the absence of major diagnostic criterion (multifocal mast cell clusters), a diagnosis of mastocytosis can still be established if three minor criteria are present. Patients with only one or two minor criteria pertaining to mast cell clonality (CD25 expression or c-kit D816V mutation) qualify for a

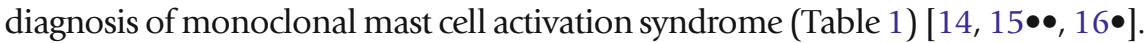
Sometimes, subdiagnostic small clusters (i.e., less than 15 cells per cluster) of spindle shaped mast cells can be visualized by tryptase immunohistochemistry, especially in perivascular and paratrabecular locations. These clusters always express the aberrant diagnostic marker of CD25 in mastocytosis.

The diagnosis of mast cell disease is straightforward in a patient with urticaria pigmentosa skin lesions. Most adult patients with urticaria pigmentosa have evidence of bone marrow involvement and systemic mastocytosis at the time of cutaneous findings. The evaluation of the patient

\section{Table 1. WHO Criteria for Systemic Mastocytosis*}

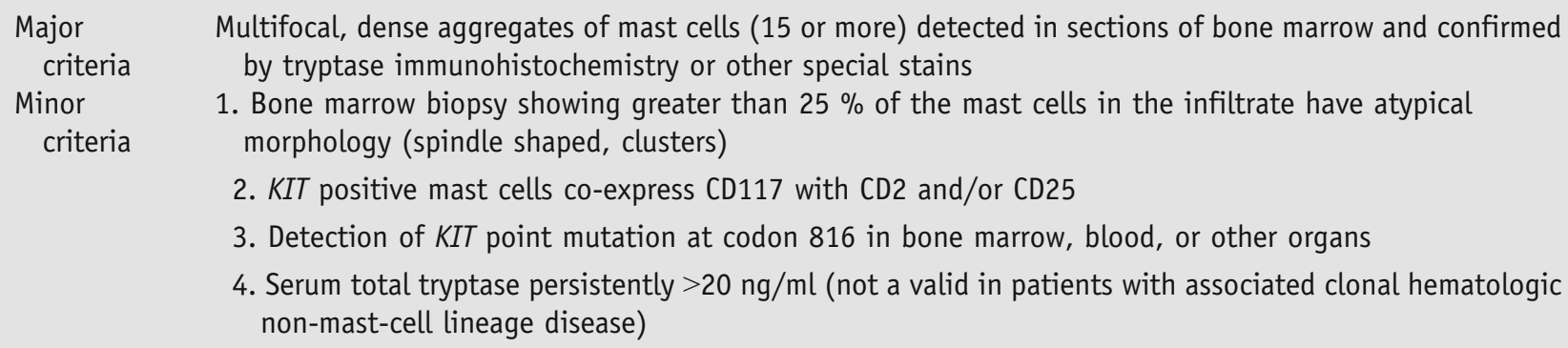


presenting with multiple recurrent anaphylactic episodes in the absence of urticaria pigmentosa is more challenging. A baseline serum tryptase level is generally obtained in these patients as a first step. One study examining patients with clonal and non-clonal mast cell activation disorders (the former including mastocytosis and monoclonal mast cell activation syndrome; and the latter idiopathic anaphylaxis) without urticaria pigmentosa skin lesions, found male gender, elevated tryptase, presence of hypotensive syncope, and lack of urticaria/angioedema as predictive factors for clonal mast cell disease. A score-based algorithm was suggested to decide which would benefit from a bone marrow examination [11]. Hymenoptera stings were the predominant trigger for anaphylaxis in patients with clonal mast cell disease, whereas drugs were implicated in patients with non-clonal disease. Increased frequency of clonal mast cell disease in patients with systemic hymenoptera reactions has also been confirmed in other studies [14] (Table 2).

Therefore we suggest starting the evaluation with a baseline tryptase level, in addition to the standard work-up, to determine an IgE mediated cause of anaphylaxis (Fig. 1). We suggest a bone marrow examination to look for markers of clonal mast cell disease in the following circumstances:

1. Baseline tryptase level $>20 \mathrm{ng} / \mathrm{ml}$ in the absence of other known causes of tryptase elevations

2. Hypotensive syncope or presyncope during anaphylactic event as opposed to hives and angioedema.

3. History of anaphylactic hymenoptera allergy and tryptase levels $>11.4 \mathrm{ng} / \mathrm{ml}$.

4. Evidence of urticaria pigmentosa on skin examination.

5. Presence of D816V c-kit mutation in peripheral blood sample.

Some European Centers suggest higher cutoffs for tryptase levels (e.g., 25$30 \mathrm{ng} / \mathrm{ml}$ ), as non-specific tryptase elevations can be seen in that range without mastocytosis. Examples of other conditions causing elevated tryptase levels include renal failure, myeloid neoplasms, parasitic disease, chronic urticarial, and idiopathic. One study suggested that urinary histamine metabolites can be useful to select patients with a high probability of having

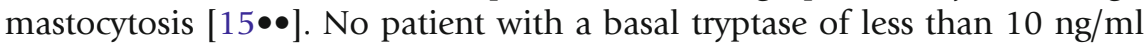

\section{Table 2. Classification of idiopathic anaphylaxis (IA)*}

\begin{tabular}{|c|c|c|}
\hline Classification & Symptoms & Frequency \\
\hline $\begin{array}{l}\text { IA-Generalized } \\
\text { infrequent }\end{array}$ & $\begin{array}{l}\text { Hypotension, gastrointestinal disruption, bronchospasm, } \\
\text { or cardiovascular collapse }\end{array}$ & $\leq 5$ episodes per year \\
\hline $\begin{array}{l}\text { IA- Generalized- } \\
\text { frequent }\end{array}$ & $\begin{array}{l}\text { Hypotension, gastrointestinal disruption, bronchospasm, } \\
\text { or cardiovascular collapse }\end{array}$ & $\begin{array}{l}6 \text { or more episodes per year, or } 2 \text { or } \\
\text { more episodes in } 2 \text { months }\end{array}$ \\
\hline $\begin{array}{l}\text { IA- Angioedema- } \\
\text { infrequent }\end{array}$ & $\begin{array}{l}\text { Angioedema or urticaria without other systemic signs of } \\
\text { anaphylaxis }\end{array}$ & $\leq 5$ episodes per year \\
\hline $\begin{array}{l}\text { IA- Angioedema- } \\
\text { frequent }\end{array}$ & $\begin{array}{l}\text { Angioedema or urticaria without other systemic signs of } \\
\text { anaphylaxis }\end{array}$ & $\begin{array}{l}6 \text { or more episodes per year, or } 2 \text { or } \\
\text { more episodes in } 2 \text { months }\end{array}$ \\
\hline
\end{tabular}




\section{Mast Cell Disorder Diagnosis Algorithm- When to consider a BM biopsy}

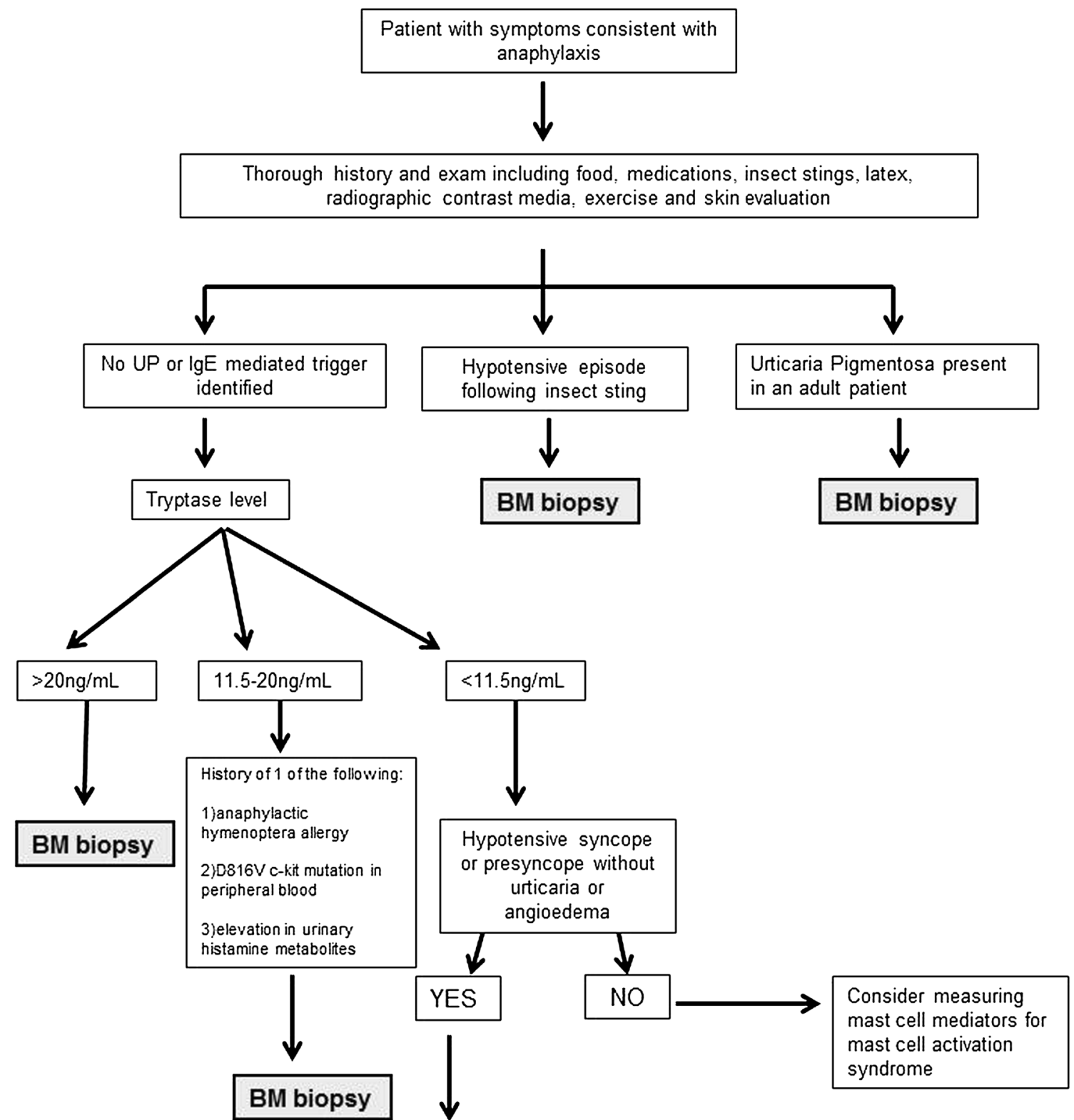

\section{BM biopsy}

\section{*BM biopsy should consist of staining for tryptase, CD117,CD25 and c-kit D816V mutation}

Fig. 1. Diagnostic algorithm in patients with a history of anaphylaxis. The first step is a thorough clinical and physical history in an attempt to identify any common triggers of anaphylaxis. Depending on the absence or presence of such factors (ex. Urticaria Pigmentosa), a bone marrow biopsy may be warranted. Further considerations regarding a bone marrow biopsy are based on the patient's tryptase level as well as other risk factors indicative of mast cell disease. 
had mastocytosis. In those with tryptase levels $>10 \mathrm{ng} / \mathrm{ml}$, those with mastocytosis had higher urinary N-methylhistamine or MIMA levels. Another approach to patients with normal or slightly elevated tryptase levels is to check D816V c-kit mutation in peripheral blood. If the mutation is detectable, a referral for bone marrow biopsy is appropriate.

The bone marrow is then subjected to evaluation for WHO criteria for systemic mastocytosis. Systemic mastocytosis is diagnosed if one major and one minor criteria, or three minor criteria are met. Patients who show either c-kit D816V mutation in peripheral blood or bone marrow, and/or CD25 staining on mast cells, without meeting other WHO criteria, are diagnosed as MMAS. Patients who do not meet any criteria are diagnosed with IA in the absence of another IgE or non-IgE mediated diagnosis.

If a diagnosis of idiopathic anaphylaxis is made, further classification can be considered. This is done according to the symptoms as well as the frequency of attacks. Greenberger and Grammer proposed classification of patients who experience six or more episodes per year or two or more episodes

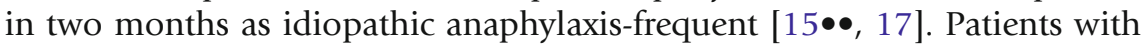
fewer episodes are classified as idiopathic anaphylaxis-infrequent. This distinction helps to determine who will require daily suppressive therapy such as prednisone. In this scheme, patients can be further characterized based on the extent of their symptoms. Those patients experiencing angioedema or urticaria, without other systemic signs of anaphylaxis, are described as idiopathic anaphylaxis-angioedema. Patients experiencing symptoms of hypotension, gastrointestinal disruption, bronchospasm, or cardiovascular collapse are termed as idiopathic anaphylaxis-generalized. This type of classification system allow patients to be divided into four groups: idiopathic anaphylaxis-generalized-infrequent; idiopathic anaphylaxis-generalized-frequent; idiopathic anaphylaxis-angioedema-infrequent; or idiopathic anaphylaxis-angioedema-frequent. Such characterization schemes help shape prognostic as well as therapeutic considerations.

\section{Prognosis}

The long term prognosis in patients with idiopathic anaphylaxis is good. The frequency of episodes rarely escalates in a particular patient [1]. Long term follow-up studies have found that over $90 \%$ of patients with frequent idiopathic anaphylaxis, and $65 \%$ with infrequent idiopathic anaphylaxis, are considered to be in remission (defined as no episodes and no corticosteroid therapy in the past year) after a few years $[18,19 \bullet]$. Recurrence after remission is also quite rare [1]. Fatalities from IA are uncommon [1] however, fatalities can occur in patients with cardiovascular or pulmonary disease, or with an underlying diagnosis of mastocytosis, especially after exposure to IgE mediated anaphylactic triggers, such as hymenoptera stings or drugs.

In general, treatment focuses on immediate treatment with self-injectable epinephrine and maintenance therapy with anti-mast cell mediator agents and immune suppression with glucocorticoids. 


\section{Pharmacologic treatment}

Patients with frequent symptoms require maintenance therapy. This therapy is aimed as prophylaxis to prevent attacks as well as therapeutic for breakthrough attacks.

- H1 blockers- An H1-antihistamine is usually the first line of treatment and is included along with prednisone in most treatment regimens. Use of a second generation H1 blocker such as fexofenadine $180 \mathrm{mg}$ or cetirizine $10 \mathrm{mg}$ once or twice daily allows for selective antagonism of peripheral histamine $\mathrm{H} 1$ receptors, while not causing sedation like first generation $\mathrm{H} 1$ blockers. H1 antihistamines should be used with caution in patients with renal insufficiency. Side effects included headache, dizziness, dyspepsia, and diarrhea. In addition to a second generation $\mathrm{H} 1$ blocker, a first generation $\mathrm{H} 1$ antihistamine may be used as needed for breakthrough symptoms. These may include hydroxyzine 25-50 mg, or diphenhydramine 25-50 mg.

The use of combination glucocorticoid/H1 blockade is recommended for patients with frequent attacks. One study showed that 19 of 22 patients had fewer attacks after the initiation of this combination of therapy. After completing this therapy, approximately $50 \%$ of patients went into remission [20].

- Glucocorticoids-A typical regimen is initiated with 40 to $60 \mathrm{mg}$ of prednisone orally per day for one week, or until the patient's symptoms have resolved. Various taper regimens can then be used. One report suggests using the same dose that induced remission every other day for two more weeks. If the patient continues to be in remission the dose is weaned by 5 to $10 \mathrm{mg}$ (still on alternate days) every two weeks. If breakthrough symptoms occur while weaning the dose, the dose should be increased to the last effective dose, and weaning may be reattempted when the symptoms have ceased to occur. Approximately $20 \%$ of patients with frequent idiopathic anaphylaxis will not tolerate weaning, and are considered to have glucocorticoid-dependent idiopathic anaphylaxis. A much more prolonged course of prednisone is required in this subset of patients. Regimens of prednisone as described above have been shown to decrease the frequency, as well as the likelihood, of recurrent life threatening events in patients with idiopathic anaphylaxis [21]. Long term side effects of glucocorticoids include adrenal insufficiency, glaucoma, diabetes mellitus, immunosuppression, osteopenia, psychosis, and a wide variety of other adverse effects. Glucocorticoids should be used with particular caution in patients with diabetes, hypertension, osteoporosis, immunocompromised patients, and the elderly. It should be avoided in patients with active infections such as varicella or measles. Because of the risk of adrenal insufficiency it requires a slow weaning prescription in these patients.

- Epinephrine- The drug of choice for acute treatment of all forms of 
anaphylaxis including idiopathic anaphylaxis. It should be administered intramuscularly at a dose of $0.3 \mathrm{mg}$ and repeated after five minutes if no significant response is observed. Epinephrine stimulates alpha and beta adrenergic receptors, resulting in vasoconstriction and bronchial smooth muscle relaxation. Because of its cardiac effects it should be used with caution in patients with hypertension, arrhythmias, and coronary artery disease. However, because it is the only drug that prevents or reverses bronchospasm and cardiovascular collapse, it has no absolute contraindications in anaphylaxis, and failure to administer epinephrine early in the course of treatment has been repeatedly implicated in anaphylaxis fatalities. Patients should administer the drug in supine position, as opposed to while sitting or standing. Palpitations, tachycardia, dizziness, and diaphoresis are frequent side effects of epinephrine administration.

- Mast cell stabilizers- Ketotifen is an H1-anthistamine and mast cell stabilizer. It has been shown in a few case reports to be effective in reducing the corticosteroid dose in corticosteroid dependent idiopathic anaphylaxis [22]. In this series, patients started ketotifen at $2 \mathrm{mg}$ three times daily and increased to $2 \mathrm{mg}$ four times daily if the patient had recurrent episodes of anaphylaxis. Ketotifen is a first generation $\mathrm{H} 1$ blocker and therefore can cause severe sedation. It currently is not available in the USA except in ophthalmologic formulations. Cromolyn sodium, another mast cell stabilizer, has a poor systemic absorption and is therefore not effective in prevention or treatment of anaphylaxis.

- Antileukotrienes- Theoretically blocking mast cell derived CysLTs could result in beneficial effects for patients with idiopathic anaphylaxis, but no convincing evidence has been reported to date to prove its efficacy in such cases. Medications targeting synthesis or receptor binding of CysLTs such as Zileuton, montelukast, or zafirlukast are frequently used in patients with asthma or mast cell disease. It is usually prescribed at bedtime at a dosage of $10 \mathrm{mg}$. They can cause worsening depression, aggressive behavior and hallucinations (mainly in children).

- Omalizumab- A monoclonal antibody directed against IgE, thus blocking its binding to mast cells and basophils and limiting the release of mediators. It has been reported on multiple occasions to be useful in the treatment of idiopathic anaphylaxis [19•, 23]. It has also been reported to be effective in eliminating anaphylactic episodes in two patients with mastocytosis who also had concurrent asthma $[24,25 \bullet]$. Omalizumab is only approved in the USA for the treatment of asthma and chronic urticaria. It is currently an extremely expensive medication and should be considered only in those patients who are refractory to standard regimens. It does carry a black box warning of anaphylaxis and thus patients require observation injections. 


\section{Pediatric considerations}

In addition to pharmacologic therapy, triggers associated with prior episodes, such as exercise, stress, extreme temperatures, and alcohol should be avoided. Medicalert jewelry should be considered.

Systemic mastocytosis or monoclonal mast cell activation is very rare in children. Therefore, we do not recommend a routine bone marrow examination in children with idiopathic anaphylaxis unless one of the following are present.

1. Presence of D816V c-kit mutation in peripheral blood

2. Tryptase levels consistently showing an increasing trend in serial measurements $>20 \mathrm{ng} / \mathrm{ml}$

3. Unexplained hepatosplenomegaly, lymphadenopathy, or abnormal CBC with diff.

The occurrence of other causes such as foods should be interrogated with great detail in this population. Dosing regimens as outlined above should be adjusted as per specific medication recommendations.

\section{Compliance with Ethics Guidelines}

\section{Conflict of Interest}

Jim Fernandez declares that he has no conflict of interest.

Cem Akin has a consultancy agreement with Novartis.

\section{Human and Animal Rights and Informed Consent}

This article does not contain any studies with human or animal subjects performed by any of the authors.

\section{References and Recommended Reading}

Papers of particular interest, published recently, have been highlighted as:

- Of importance

- Of major importance

1. Ditto AM, Harris KE, Krasnick J, et al. Idiopathic anaphylaxis: a series of 335 cases. Ann Allergy Asthma Immunol. 1996;77:285.

2. Patterson R, Hogan MB, Yarnold PR, Harris KE. Idiopathic anaphylaxis: an attempt to estimate the incidence in the USA. Arch Intern Med. 1995;155:869-71.

3. Brown AFT, McKinnon D, Chu K. Emergency department anaphylaxis: a review of 142 patients in single year. J Allergy Clin Immunol. 2001;108:861-6.

4. Tejedor Alonso MA, Sastre Dominguez J, SánchezHernández JJ, PérezFrances C, Hoz de la Caballer B.

Clinical and functional differences among patients with idiopathic anaphylaxis. J Investig Allergol Clin Immunol. 2004;14(3):177-86.

5. Bacal E, Patterson R, Zeiss CR. Evaluation of severe (anaphylactic) reactions. Clin Allergy. 1978;8:295304.

6. Grammer LC, Shaughnessy MA, Harris KE, Goolsby CL. Ann Allergy Asthma Immunol. 2000;85(5):36871.

7. Akin C, Scott LM, Kocabas CN, et al. Demonstration of an aberrant mast-cell population with clonal 
markers in a subset of patients with "idiopathic" anaphylaxis. Blood. 2007;110:2331.

8. Castells M. Mast cell mediators in allergic inflammation and mastocytosis. Immunol Allergy Clin N Am. 2006;26:465-85.

9. Greenhawt M, Akin C. Mastocytosis and allergy. Curr Opin Allergy Clin Immunol. 2007;7:387-92.

10. Koterba A, Akin C. Differences in the clinical presentation of anaphylaxis in patients with indolent systemic mastocytosis (ISM) versus idiopathic anaphylaxis (IA). JACI. 2008;121:S68-9.

11. Alvarez-Twose I, González de Olano D, SánchezMuñoz L, Matito A, Esteban-López MI, Vega A, et al Clinical, biological, and molecular characteristics of clonal mast cell disorders presenting with systemic mast cell activation symptoms. J Allergy Clin Immunol. 2010;125(6):1269-78.

12. Agostoni A, Cicardi M. Hereditary and acquired C1-inhibitor deficiency: biological and clinical characteristics in 235 patients. Medicine (Baltimore). 1992;71:206.

13.• Valent P, Akin C, Arock M, Brockow K, Butterfield JH, Carter MC, et al. Definitions, criteria and global classification of mast cell disorders with special reference to mast cell activation syndromes: a consensus proposal. Int Arch Allergy Immunol. 2012;157(3):215-25.

This article was written by the world leaders in the mast cell field. It now includes a consensus definition and criteria to define mast cell disease as well as a helpful algorithm to aid in classifications.

14. Bonadonna P, Perbellini O, Passalacqua G, Caruso B, Colarossi S, Dal Fior D, et al. Clonal mast cell disorders in patients with systemic reactions to Hymenoptera stings and increased serum tryptase levels. J Allergy Clin Immunol. 2009;123(3):680-6.

15.• van Doormaal JJ, van der Veer E, van Voorst Vader PC, Kluin PM, Mulder AB, van der Heide S, et al. Tryptase and histamine metabolites as diagnostic indicators of indolent systemic mastocytosis without skin lesions. Allergy. 2012;67:683-90.

This article discusses the importance of specific diagnostic tools including bone marrow biopsy in patients with risk factors of mastocystosis. A helpful algorithm is provided to help guide clinical decision making.
16. Lee MJ, Akin C. Mast cell activation syndromes. Ann Allergy Asthma Immunol. 2013;111(1):5-8. This report clarifies the criteria for mast cell activation syndrome (MCAS). This diagnosis is frequently missed or incorrectly labeled. Many of these patients would benefit from a bone marrow biopsy.

17. Patterson R, Stoloff RS, Greenberger PA, Grammer LC, Harris KE. Algorithms for the diagnosis and management of idiopathic anaphylaxis. Ann Allergy. 1993;71(1):40-4.

18. Greenberger PA. Idiopathic anaphylaxis. Immunol Allergy Clin North Am. 2007;27:273.

19. Lieberman PL. Idiopathic anaphylaxis. Allergy Asthma Proc. 2014;35(1):17-23.

This concise review of the literature updates the reader on the current understanding of idiopathic anaphylaxis. Focus is placed on advancements such as mast cell disease and IgE to galactose-alpha-1, 3-galactose.

20. Krasnick J, Patterson R, Harris KE. Idiopathic anaphylaxis: long-term follow-up, cost, and outlook. Allergy. 1996;51(10):724-31.

21. Boxer MB, Greenberger PA, Patterson R. The impact of prednisone in life threatening idiopathic anaphylaxis: reduction in acute episodes and medical costs. Ann Allergy. 1989;62(3):201-4.

22. Wong S, Patterson R, et al. Efficacy of ketotifen in corticosteroid-dependent idiopathic anaphylaxis. Ann Allergy. 1991;67:359-64.

23. Babu KS, Polosa R, Morjaria JB. Anti-IgE-emerging opportunities for Omalizumab. Expert Opin Biol Ther. 2013;13(5):765-77.

24. Carter MC, Robyn JA, Bressler PB, Walker JC, Shapiro GG, Metcalfe DD. Omalizumab for the treatment of unprovoked anaphylaxis in patients with systemic mastocytosis. J Allergy Clin Immunol. 2007;119(6):1550-1.

25. Incorvaia $C$, Mauro $M$, Russello $M$, Formigoni $C$, Riario-Sforza GG, Ridolo E. Omalizumab, an antiimmunoglobulin E antibody: state of the art. Drug Des Dev Ther. 2014;8:197-207.

This article reviews Omalizumab and discusses its use and success for a variety of allergic diseases including anaphylaxis and mast cell disease. 